\title{
The subchondral bone: a new frontier in articular cartilage repair
}

\author{
Henning Madry
}

Published online: 3 February 2010

(C) Springer-Verlag 2010

In the past decades, considerable advances have been made to treat defects of the articular cartilage. Yet the problem of lesions that extend deep in the underlying subchondral bone has not received adequate attention. Subchondral bone damage is mainly associated with four individual etiologies, including traumatic osteochondral fracture, osteochondritis dissecans, osteonecrosis, and osteoarthritis. It is largely because of these very different origins of osteochondral defects that their treatment remains complicated.

In Mondorf-les-Bains, Luxembourg, from 8 to 10 October 2009, a group of researchers and physicians at the forefront of their field met to review and summarize the current state and potential future research directions of the subchondral bone in articular cartilage repair. This conference "The subchondral bone in articular cartilage repair" was co-chaired by the author and by Dr. Romain Seil (Luxembourg). The Deutsche Forschungsgemeinschaft (DFG) of Germany and the Fonds National de la Recherche (FNR) of Luxembourg jointly supported this endeavor. In this issue of Knee Surgery, Sports Traumatology, and Arthroscopy, the results of that meeting are presented as four individual review articles. They reflect not only a large body of current research advancement but also aim at identifying issues that still need to be answered.

An initial article "Basic science of the subchondral bone" [2] reviews the anatomy, morphology, physiology, and pathology of the subchondral bone in the context of articular cartilage repair and in the light of the four

\footnotetext{
H. Madry $(\square)$

Institute for Experimental Orthopaedics and Department of Orthopaedic Surgery, Saarland University Medical Center, Saarland University, Homburg, Germany e-mail: hmad@hotmail.com
}

different diseases. Evidence was shown that collagen fibrils cross the tidemark - the line that separates the calcified cartilage from the noncalcified cartilage-resulting in a strong link between these two zones. This is of clinical relevance-emphasizing the need for a meticulous removal of all calcified articular cartilage when a cartilage defect is debrided prior to a marrow-stimulating technique or autologous chondrocytes transplantation to allow for a good attachment of the repair tissue to the subchondral bone plate.

The second article provides insights into the epidemiology and imaging of the subchondral bone [3]. Osteochondral defects, account for about $5 \%$ of all articular cartilage lesions, are predominantly located on the medial femoral condyle and patella and frequently associated with lesions of the menisci or the anterior cruciate ligament. Magnetic resonance imaging is the key procedure to evaluate articular cartilage and subchondral bone. However, when choosing an imaging technique to investigate osteochondral defects, the nature of the disease that causes the subchondral bone lesion has to be taken into account. For example, bone scintigraphy is valuable for early diagnosis of spontaneous osteonecrosis about the knee, while CT arthrography is more useful to evaluate the stability of the osteochondral fragment in osteochondritis dissecans.

Another article is devoted to disease-specific clinical problems associated with the subchondral bone: osteochondral fracture, osteochondritis dissecans, osteonecrosis, and osteoarthritis [4]. If left untreated, all of them have the potential to progress from a prearthrotic deformity to joint destruction. Size and location of the subchondral lesion, stage of the disease, age of the patient, and congruency of the joint strongly influence the result of the surgical therapy. 
The last article of this series delves into specific questions of contemporary approaches to common surgical problems affecting the subchondral bone [1]. Issues that continue to complicate the current treatments are reviewed, such as the thickening of the subchondral plate and formation of intralesional osteophytes observed after marrow stimulation techniques, or the large osteochondral defects resulting from removal of focal knee resurfacing implants.

Altogether, these findings emphasize the need for a specific knowledge and comprehension of each type of subchondral bone lesion and its natural history as the key for selecting the optimal therapy for our patients. After many decades of focusing our main interest on the articular cartilage, it is gradually becoming clear that integrity of the subchondral bone is the key for preserving the function of the entire osteochondral unit. A better understanding of the basic science and pathophysiology of the subchondral region, together with additional investigations in animal models and patients will probably translate into improved strategies for the repair of cartilage defects that arise from or extend into the subchondral bone.

We hope readers will enjoy these contributions.

\section{References}

1. Gomoll A, Madry H, Knutsen G, van Dijk CN, Seil R, Brittberg M, Kon E (2010) The subchondral bone in articular cartilage repaircurrent problems in the surgical management. Knee Surg Sports Traumatol Arthrosc 18. doi:10.1007/s00167-010-1072-x

2. Madry H, van Dijk CN, Mueller-Gerbl M (2010) The basic science of the subchondral bone. Knee Surg Sports Traumatol Arthrosc 18. doi:10.1007/s00167-010-1054-z

3. Menetrey J, Unno-Veith F, Madry H, van Breuseghem I (2010) Epidemiology and imaging of the subchondral bone in articular cartilage repair. Knee Surg Sports Traumatol Arthrosc 18. doi: 10.1007/s00167-010-1053-0

4. Pape D, Filardo G, Kon E, van Dijk CN, Madry H (2010) Diseasespecific clinical problems associated with the subchondral bone. Knee Surg Sports Traumatol Arthrosc 18. doi:10.1007/s00167010-1052-1 